\title{
Gas-Phase Formation of Large Neutral Alkaline-Earth Metal Tryptophan Complexes
}

\author{
Markus Marksteiner, Philipp Haslinger, Hendrik Ulbricht, \\ Michele Sclafani, Harald Oberhofer, Christoph Dellago, and \\ Markus Arndt \\ Faculty of Physics, University of Vienna, Vienna, Austria
}

We report on the first observation of isolated large neutral metal amino acid complexes such as $\operatorname{Trp}_{n} \mathrm{Me}_{k}$, with $\mathrm{Me}=\mathrm{Ca}, \mathrm{Ba}, \mathrm{Sr}$, cluster combinations covering $n=1 \ldots .33, k=0.2$ and masses beyond $6500 \mathrm{u}$. The cluster beam is generated using UV laser desorption from a mixed powder of alkaline-earth metal salts and tryptophan inside a cluster mixing channel. The particles are detected using VUV photoionization followed by time-of-flight mass spectroscopy. The enhanced stability of metal amino acid clusters over pure amino acid clusters is substantiated in molecular dynamics simulations by determining the gain in binding energy related to the inclusion of the metal atoms. (J Am Soc Mass Spectrom 2008, 19, 1021-1026) (C) 2008 American Society for Mass Spectrometry

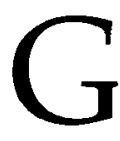
as-phase investigations of organic molecules have received an enormous and still increasing attention since the successful establishment of various laser desorption [1-3] and electrospray ionization techniques [4]. The majority of studies so far focused on the generation and use of ionic species. Our work, presented here, focuses on the gas-phase formation of neutral biomolecular clusters as did already some very early studies in the field [1]. Such experiments are often motivated by the desire to better identify the role of solvation and desolvation for the relative importance of various binding forces in organic clusters and molecules [5].

The particle's neutrality is also an important criterion in efforts to establish new beam methods for quantum interferometry with molecular matter waves [6-9] as well as for precision metrology on large molecules [10-12]. Neutral molecules are much better isolated from any electromagnetic environment and they may therefore propagate coherently, without noticeable perturbation, even in a rather noisy field environment.

Biomolecular clusters are exciting study objects for future matter wave experiments as they permit one to scale the mass in well-defined steps, while at the same time changing the internal properties in an intriguing way: the number of possible conformations as well as the number of allowed configurational and vibrational transitions scales exponentially with the size of the cluster [13], and it is still an open question how this will affect de Broglie interferometry [14], which formally considers only the center of mass motion alone.

Address reprint requests to Professor M. Arndt, Faculty of Physics, University of Vienna, Boltzmanngasse 5, 1090 Vienna, Austria. E-mail: markus.arndt@ univie.ac.at
The use of neutral molecules requires the availability of a suitable ionization mechanism, if one also wants to exploit common mass spectroscopy methods for characterizing the desorbed species. Ionization methods used in the past have been as diverse as the molecules under investigation: UV multi-photon ionization [1] was in competition with single-photon ionization using VUV lamps [15], VUV lasers [16], dye tagging of molecules [17], femtosecond ionization [18], or even thermionic emission of electrons [19]. But it has still remained a challenge to find reproducible methods for photoionizing biomolecules and complexes with masses exceeding $3000 \mathrm{u}[17,20,21]$.

The formation and photo-detection of small amino acid [22] and nucleotide clusters [23], both hydrated and solvent-free, were already reported for objects up to $1400 \mathrm{u}$. Ultracold small bioclusters can be formed by pick-up in helium nano-droplets [24]. Complexes formed between metal atoms and amino acids were also studied to understand their structure [25], their fragmentation pathways [26, 27], and metal ion affinities [28].

Here we extend these investigations to much larger organometallic complexes composed of up to more than 30 tryptophan molecules with masses exceeding $6500 \mathrm{u}$, that can still be detected using VUV photoionization and time-of-flight mass spectrometry.

\section{Experimental}

We generate the molecular macroclusters using pulsed laser evaporation and collisional cooling: the pulsed beam of a Nd:YAG laser (Quantel Brilliant $\lambda=355 \mathrm{~nm}, \tau=$ $5 \mathrm{~ns}, E_{d}<20 \mathrm{~mJ}$ ) is focused with a spot size of about 700 $\mu \mathrm{m}$ diameter onto the sample rod that is covered with a layer of pressed molecular powder (see Figure 1). 


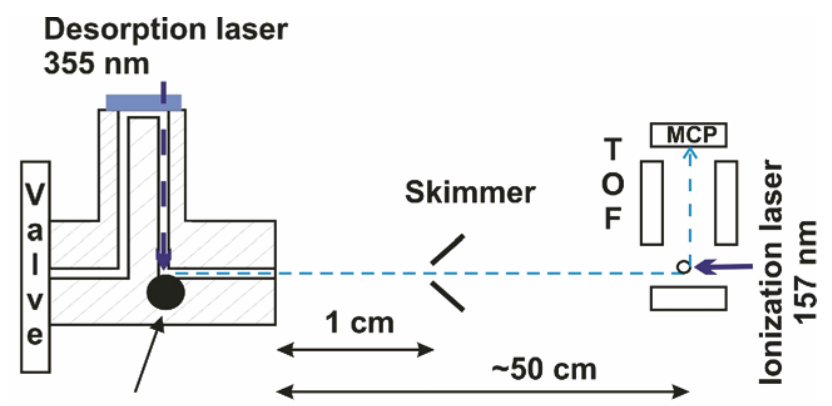

Desorption target

Figure 1. Beam generation and detection. Pulsed UV radiation ablates molecules from a rotating sample rod, which is covered by an amino acid metal powder mixture. The valve releases a short noble gas pulse, which entrains the desorbed molecules inside the mixing channel. The channel is formed such as to purge and clean the laser transmission window with each gas pulse. The molecules pass a skimmer on their way to the high-vacuum chamber, where they are exposed to VUV photoionization and time-of-flight mass spectrometry (TOF-MS).

A fast valve (Parker General Valve 99), with a 0.5 mm exit nozzle and an opening time of less than $500 \mu \mathrm{s}$ releases a noble gas pulse into the mixing channel to cool the molecules while they are desorbed. The gas backing pressure is usually limited to about 2 bar. The target is placed inside the channel to allow for efficient collisional cooling of the desorption plume. The molecules cluster in the presence of the buffer gas. They are then entrained by the carrier flow which expands as a supersonic jet behind the channel orifice.

The clusters traverse a $1 \mathrm{~mm}$ skimmer into the high vacuum chamber, where they are detected, about $50 \mathrm{~cm}$ behind the mixing channel. Postionization of the neutral amino acids and complexes is achieved with pulsed VUV radiation at $157 \mathrm{~nm}$, derived from a $\mathrm{F}_{2}$ excimer laser (Coherent Existar, $5 \mathrm{~ns}, \mathrm{E},<3 \mathrm{~mJ}$ ). The emerging cluster ions are mass selected and registered in an orthogonal time-of-flight mass spectrometer (Kaesdorf) with a mass resolution of $m / \Delta m>500$.

The experiment is running at a repetition rate of 10 Hz. Thirty to 50 desorption shots are usually averaged per mass spectrum. The mass scale can be calibrated with tryptophan, $\beta$-carotene and gramicidin D.

To form organometalic complexes, we mix the alkaline earth metal salt $\mathrm{CaCO}_{3}$ with D-tryptophan, $\mathrm{C}_{11} \mathrm{H}_{12} \mathrm{~N}_{2} \mathrm{O}_{2}$ with $m=204.23 \mathrm{u}$, and cellulose, $\left(\mathrm{C}_{6} \mathrm{H}_{10} \mathrm{O}_{5}\right)_{n}$, in a weight ratio of 1:5:5. This yields on average 2 calcium atoms per 5 tryptophan molecules in the sample. All molecules were used as purchased (Sigma Aldrich, Vienna, Austria) without any further purification. The molecular powder is pressed onto a fine-threaded metal rod. This screw is rotated during laser desorption to gradually refresh the molecule surface that is exposed to the laser shots.

Our setup is sensitive to neutral clusters which propagate freely to the ionization region half a meter downstream. Charged clusters remain undetected because of the beam geometry as confirmed by the absence of a signal without post-ionization.

\section{Results}

When we load the tryptophan-cellulose substrate with $\mathrm{CaCO}_{3}$ we observe the formation of unusually large neutral organometallic complexes as shown in Figure 2. They contain a large number of tryptophan molecules and usually a single, occasionally also two calcium atoms per tryptophan complex. $\operatorname{Trp}_{33} \mathrm{Ca}_{1}$ with $m=$ $6,779 \mathrm{u}$ was the largest cluster that was observed. This is even more massive than insulin which we could not post-ionize in our setup-neither with radiation at 157 $\mathrm{nm}$ up to $3 \mathrm{~mJ}$ nor at $266 \mathrm{~nm}$ up to $30 \mathrm{~mJ}$.

The relative abundance of clusters is influenced by various parameters during their formation: the energy and focal width of the desorption laser, its peak intensity, the nozzle temperature, the time delay between desorption and cooling gas injection, the refreshing rate for the exposed molecular surface and more [29]. Also the detection parameters influence the observed cluster distribution: the ionization laser energy determines the probability for the absorption of a second photon by the already ionized complex. This added heating can provide excess energy and induce post-ionization fragmentation as well as the preferential appearance of smaller cluster ions. We see indications of fragmentation at elevated ionization energies and cluster masses beyond $3000 \mathrm{u}$. The post-acceleration voltage of the time-offlight mass spectrometer (TOF) is limited to $18 \mathrm{kV}$. It determines the secondary electron yield in the multichannel plate at the TOF's exit and probably sets the most stringent upper experimental constraint to the maximal observed cluster mass [30].

We have extended our investigation to other alkalineearth metals to identify more clearly the number of metal atoms that are required to bind a large amino acid complex. Similarly to the calcium experiments we now mix the metal carbonates $\mathrm{CaCO}_{3}, \mathrm{SrCO}_{3}$ and $\mathrm{BaCO}_{3}$ in a

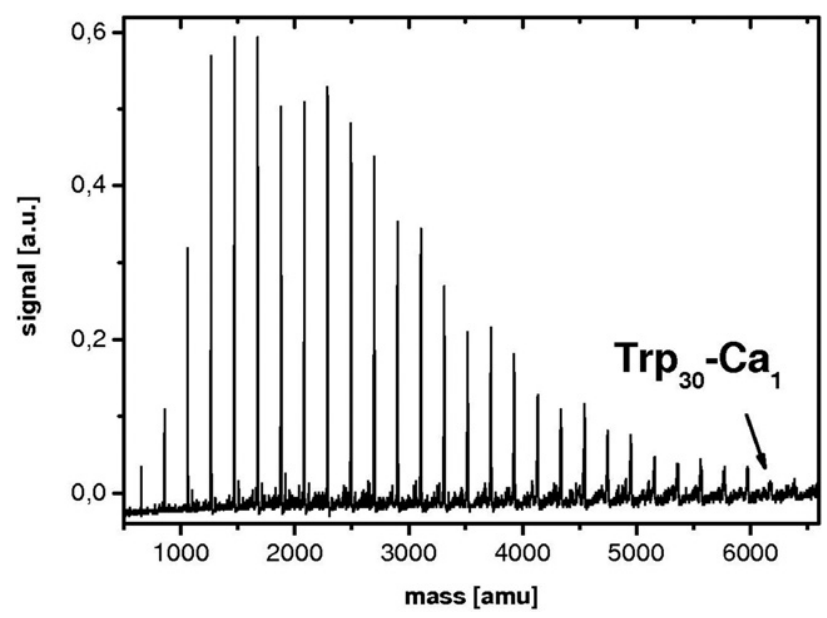

Figure 2. $\operatorname{Trp}_{\mathrm{k}} \mathrm{Ca}_{\mathrm{n}}$ clusters form at an argon backing gas pressure of $2 \mathrm{bar}$ and a nozzle temperature of $300 \mathrm{~K}$. All complexes in this distribution preferentially contain a single calcium atom $(\mathrm{k}=1)$ even when the cluster grows to more than $n=30$ tryptophan molecules. 
weight ratio of 1:1.48:1.97 to obtain the same number density of all metal salts. The metal carbonate powder is mixed to tryptophan and cellulose in a weight ratio of 2.8:8:5. The observed cluster distribution is then determined by various factors related to cluster formation and detection. The cluster formation is influenced first by the release of the metal atom from the salt (laser energy, intensity and wavelength), second by the binding probability of the metal to a single tryptophan. Also the changing polarizability and polarity assist in attracting and binding further tryptophan molecules. The detection efficiency is both determined by the ionization step and by the secondary electron multiplication efficiency inside the TOF. The ionizing laser pulse has an energy of $7.89 \mathrm{eV}$, which is very close to the single-photon ionization threshold of some tryptophan configurations. The metal atom will modify these energies and even do this in dependence of the particular cluster and molecule configuration. This in turn depends also on the molecular temperature and therefore also on the source conditions. The experimental data are shown in Figure 3.

In this direct comparison the most intense fraction of the molecular beam contains strontium as a seed atom. The ionization potentials or electron affinities of all three alkaline-earth metals can not yet explain this behavior. It might be related to the carbonate dissociation efficiency. For all three cluster types the inclusion and detection of a single metal atom is clearly the most probable process.

\section{Discussion}

It is interesting to note that although the desorbed powder contains about as many bound metal atoms as tryptophan molecules, we never observe any significant signal of pure metal clusters. This is in good agreement with the assumption that metal atoms will appear

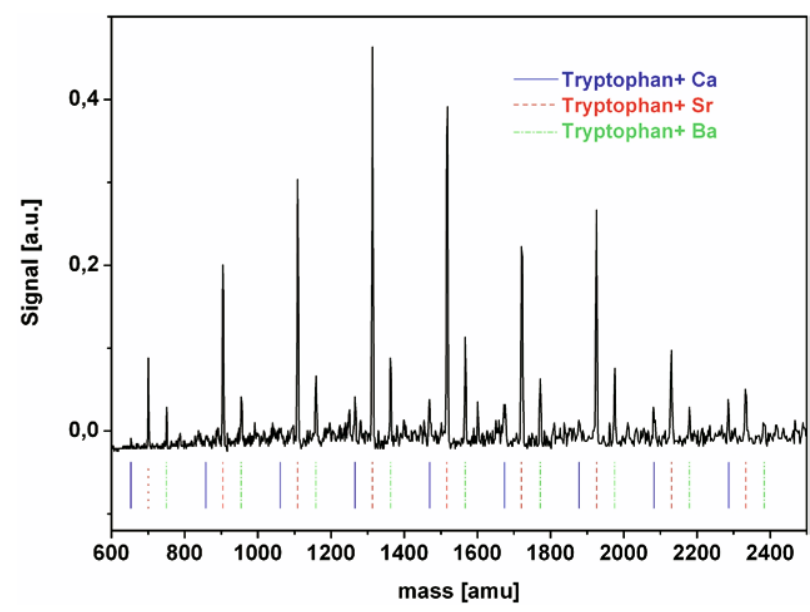

Figure 3. Comparison of metal tryptophan complex formation when three different alkaline-earth metal salts- $-\mathrm{CaCO}_{3}, \mathrm{SrCO}_{3}$, and $\mathrm{BaCO}_{3}$-are mixed to the biomolecular powder with equal number concentrations of the metal atoms. predominantly as charged species when they are isolated from their carbonate ion and positively charged ions would neither bind with each other nor be detected in our setup.

Even more interestingly, we note that pure tryptophan clusters are also preferentially suppressed at elevated temperatures, i.e., at about room temperature in our present experiments. Figure 2 shows that each individual peak corresponds to an integer number of tryptophan molecules associated with a single calcium atom. Metal-free compounds are completely absent in this experiment, and we have seen first indications that the absence or presence of metal atoms shows a strong temperature dependence.

To shed some light on this observation we have simulated various sizes of both pure $\operatorname{Trp}_{n}$ and $\operatorname{Trp}_{n} \mathrm{Ca}_{1}$ clusters, respectively, in an equilibrium molecular dynamics simulation. These calculations are carried out for individual clusters and do neither describe their formation nor their growth. Therefore, results obtained from these simulations yield only partial information on the experimental cluster size distributions. All simulations were performed using the NAMD [31] package with the CHARMM $2.7[32,33]$ force field. The thermalizing effect of the carrier gas was taken into account by assuming that the system evolves according to the Langevin equations of motion in which a noisy environment of a given temperature is coupled to the system and acts as a thermostat.

To simulate the $\operatorname{Trp}_{n} \mathrm{Ca}_{1}$ clusters a modification of the CHARMM force field was necessary. A comparison of the experimental Ca-ionization energies with the tryptophan electron affinity suggests that a neutral calcium atom is easily ionized by a Trp molecule. Since, as demonstrated by our experiments, each TrpCa cluster is neutral as a whole, we assume that the doubly positive calcium ion is neutralized by negative charges located on the Trp-molecules. More specifically, we model the clusters as consisting of a $\mathrm{Ca}^{2+}$ ion, $(k=N-2)$ neutral Trp-molecules, and two ionized Trp-molecules each carrying a single negative charge. For these negatively charged molecules, we modify the atomic charges included in the CHARMM force field to account for the additional electron. Density functional theory calculations of the lowest unoccupied molecular orbital carried out with the CPMD package [34] using the BLYP functional with a cutoff of 80 Rydberg indicate that the extra electron is delocalized over three carbon atoms in the aromatic rings of the tryptophan molecule. Charges of corresponding magnitude are added to the CHARMM force field at these positions.

In our simulations, we first thermalize both pure and calcium containing clusters for a time of $1 \mathrm{~ns}$ at $300 \mathrm{~K}$. Then, the time evolution of the cluster is followed for 9 ns during which averages are collected. Snapshots of various clusters taken from our simulations are shown in Figure 4. For all cluster sizes larger than $n=2$, both the clusters with and without calcium have a rather compact shape. 
To assess stability and shape of the clusters quantitatively, we calculate the equilibrium average radii of gyration and binding energies, both depicted in Figure 5 versus the cluster size $n$. The radii of gyration for the clusters with and without calcium are roughly the same for all cluster sizes confirming the visual impression gleaned from the simulations that shapes and sizes of pure and metal containing clusters do not differ significantly. Note that the large error bars for the smaller pure clusters stem from the fact that they partly dissociated during our simulation runs due to their rather small binding energies. These near dissociation events, which occur due to the much smaller binding energies of the metals free clusters, also cause the average radii of gyration to be larger in these cases. Simulations carried out at lower temperatures yielded very similar results.

Binding energies as a function of the cluster sizes are depicted in the bottom panel of Figure 5. Here, the binding energy is defined as the energy necessary to detach a single tryptophan molecule from the cluster

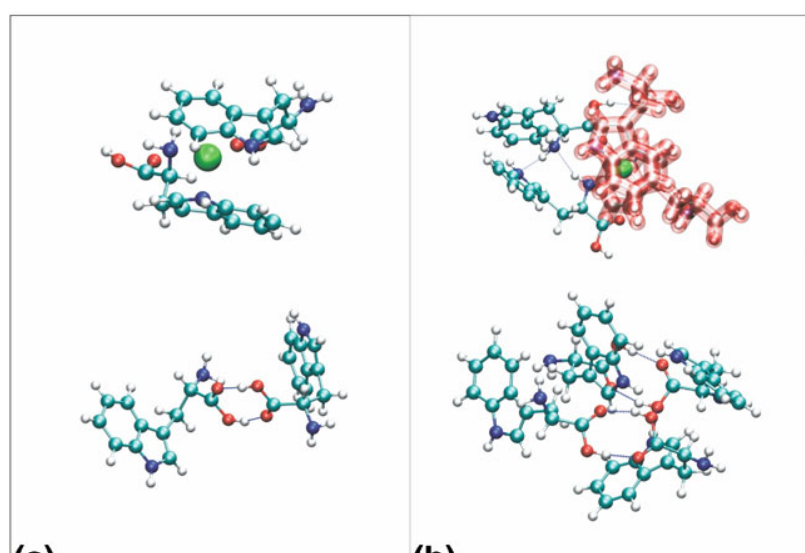

(a)

(b)
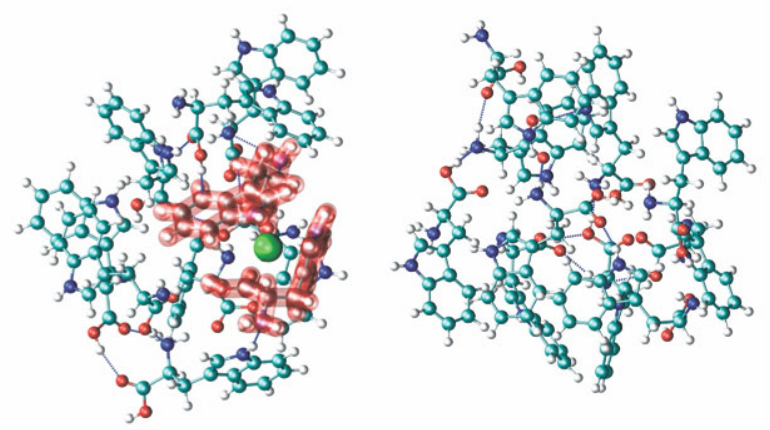

(c)

Figure 4. Snapshots from molecular dynamics simulations of $n=2(\mathrm{a}), n=4$ (b) and $n=10$ (c) tryptophan molecules with and without calcium. The calcium atom is depicted as a green large sphere and the two charged Trp molecules are highlighted in shaded red. Note that the charged tryptophans are bound rather tightly to the metal by electrostatic forces, while the rest of the cluster is mainly stabilized by hydrogen bonds (dashed blue lines) and van der Waals interactions.

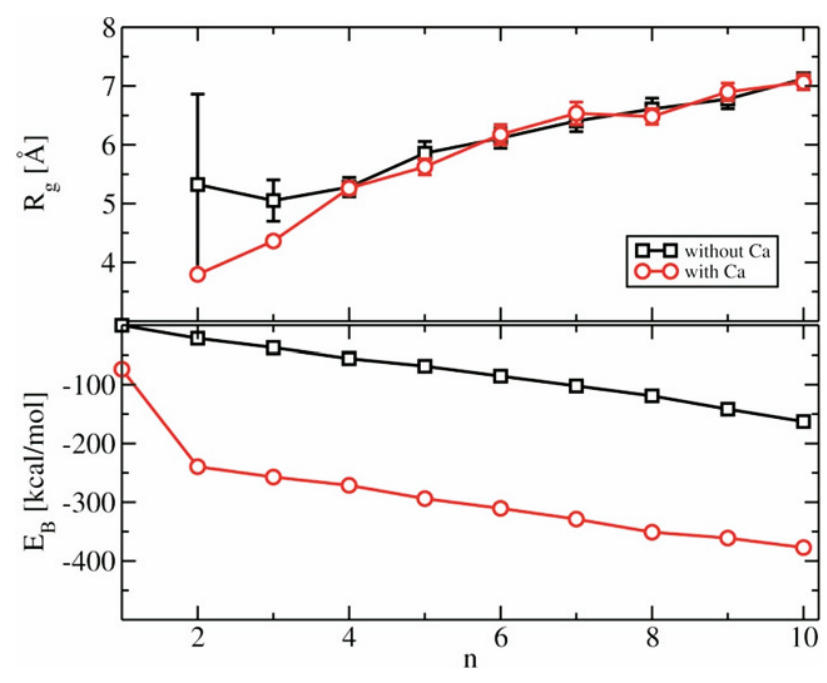

Figure 5. Radii of gyration (top) and binding energies (bottom) for $\operatorname{Trp}_{n} \mathrm{Ca}_{1}$ complexes as a function of the number of Trp-molecules.

and move it towards infinity. For the determination of the binding energies, we compute the average potential energy for all cluster sizes $n$ and subtract, for the metal containing clusters, $n-2$ times the average energy of a single uncharged tryptophan and twice the average energy of a charged Trp. For the pure clusters, we simply subtract $n$ times the potential energy of an uncharged tryptophan. Additionally, we correct for the fact that on removal of a charged tryptophan it is energetically more favorable for the system to return to its uncharged state, by adding the charge-transfer energy difference to the binding energy.

In agreement with the experiments, the calcium containing clusters are energetically strongly stabilized with respect to the pure ones. Most of the increased binding energy, however, is due to the two charged Trp-molecules binding to the calcium ion. Adding more tryptophans beyond the first two lead to nearly the same energetic gain both for the cluster with and without metal as reflected in the identical slope of the two energy curves shown in Figure 5. One might conceive of different growth mechanisms for large clusters: on the one hand they could aggregate from preformed clusters or accumulate around a single nucleus. The energy diagram indicates that the formation of many small preformed clusters, such as $\operatorname{Trp}_{2} \mathrm{Ca}_{1}$ would be energetically favorable. However, we find in all experiments only a single metal atom, and very rarely two, per cluster. This holds even for clusters composed of more than 30 amino acids. This strongly supports a second view, namely that the amino acids agglomerate one by one around a single $\operatorname{Trp}_{2} \mathrm{Ca}_{1}$ nucleation core. A tentative explanation for that is based on the prevalence of polarization effects around the neutral $\operatorname{Trp}_{2} \mathrm{Ca}_{1}$ core, which possesses a strong charge asymmetry. This will tend to orient the surrounding amino acid dipoles. It may also enhance the oriented dipole mo- 
ments through the interaction with the tryptophan's static scalar polarizability, which amounts to about 20 $\AA^{3}$ [35]. Note, however, that the empirical force field used here does not include an electronic polarizability and thus the latter effect is neglected in our calculations.

While the monotonically decreasing binding energies shown in Figure 5 seem to favor the formation of large clusters, entropic effects neglected in our purely energetic calculation tend to destabilize larger clusters. A more detailed description of the cluster size distribution would also require taking into account the nonequilibrium kinetics of the cluster formation and growth process. While modeling the cluster distribution during the formation period in the hot, dense, and rapidly expanding laser desorption plume seems almost prohibitively complex, a simpler near-equilibrium treatment based on kinetic equations may be possible during the much longer dwell time of about $100 \mu \mathrm{s}$ inside the room temperature collision channel. The subsequent co-expansion into the high-vacuum chamber, together with the Argon carrier gas, will lead to an additional cooling and further stabilization and possibly further aggregation of the clusters. The substantial increase in cluster number and size with the addition of a collision channel to a free jet design supports, however, the hypothesis that the majority of all clustering events will occur inside the mixing channel. Efforts to model the time evolution of the cluster size distribution eventually terminated by the supersonic jet expansion are underway in our laboratory.

\section{Conclusions}

We have observed the formation and single-photon ionization of very large alkaline-earth metal amino acid complexes. Numerical simulations substantiate the observation that metal amino acid complexes formed under near room-temperature conditions are more stable and more easily detected than their metal-free analogs. The presence and detectability of these unusually large compounds is expected to be of importance for future experiments in molecule interferometry and metrology [11] as it allows one to gradually tune the cluster mass with elements that are much more intricate and variable than pure metal or semiconductor clusters alone. The gradual addition of water vapor in future experiments shall also allow us to explore the stability of giant metal amino acid clusters in a solvating biomimetic environment [36].

\section{Acknowledgments}

The authors acknowledge support for this work by the Austrian Science Funds FWF within the programs START Y177-2 and SFB F1505, and within the Science College "Computational Materials Science" under grant W004, as well as by the Italian Fondazione Angelo Della Riccia.

\section{References}

1. Grotemeyer, J.; Bosel, U.; Walter, K.; Schlag, E. W. A General Soft Ionization Method for Mass Spectrometry: Resonance Enhanced MultiPhonon Ionization of Biomolecules. I. Am. Chem. Soc. 1986, 21, 645-653.

2. Tanaka, K.; Waki, H.; Ido, Y.; Akita, S.; Yoshida, Y.; Yoshida, T.; Matsuo T. Protein and Polymer Analyses up to $m / z 100,000$ by Laser Ionization Time-of-Flight Mass Spectrometry. Rapid Commun. Mass Spectrom. 1988, 2, 151-153.

3. Karas, M.; Hillenkamp, F. Laser Desorption Ionization of Proteins with Molecular Mass Exceeding 10,000 Daltons. Anal. Chem. 1988, 60, 2299 2301.

4. Fenn, J. B.; Mann, M.; Meng, C. K.; Wong, S. F.; Whitehouse, C. M. Electrospray Ionization for Mass Spectrometry of Large Biomolecules. Science 1989, 246, 64-71.

5. Schalley, C. A. Molecular Recognition and Supramolecular Chemistry in the Gas Phase. Mass Spectrom. Rev. 2001, 20, 253-309.

6. Arndt, M.; Nairz, O.; Voss-Andreae, J.; Keller, C.; G. van der Zouw, G.; Zeilinger, A. Wave-Particle Duality of C60 Molecules. Nature 1999, 401 680-682.

7. Hackermüller, L.; Uttenthaler, S.; Hornberger, K.; Reiger, E.; Brezger, B.; Zeilinger, A.; Arndt, M. Wave Nature of Biomolecules and Fluorofullerenes. Phys. Rev. Lett. 2003, 91, 90408-90412.

8. Gerlich, S.; Hackermüller, L.; Hornberger, K.; Stibor, A.; Ulbricht, H.; Gring, M.; Goldfarb, F.; Savas, T.; Müri, M.; Mayor, M.; Arndt, M. A Kapitza-Dirac-Talbot-Lau interferometer for highly polarizable molecules. Nat. Phys. 2007, 3, 711-715.

9. Marksteiner, M.; Kiesewetter, G.; Hackermüller, L.; Ulbricht, H.; Arndt, M. Cold Beams of Biomolecules for Quantum Optics. Acta Phys. Hung. 2007, B 26/12, 87-94.

10. Antoine, R.; Dugourd, P.; Rayane, D.; Benichou, E.; Broyer, M.; Chandezon, F.; Guet, C. Direct Measurement of the Electric Polarizability of Isolated C60 Molecules. J. Chem. Phys. 1999, 110, 9771-9772.

11. Berninger, M.; Stefanov, A.; Deachapunya, S.; Arndt, M. Polarizability Measurements in a Molecule Near-Field Interferometer. Phys. Rev. A 2007, 76, 013607-013611.

12. Deachapunya, S.; Fagan, P. J.; Major, A. G.; Reiger, E.; Ritsch, H. Stefanov, A.; Ulbricht, H.; Arndt, M. Slow Beams of Massive Molecules. Eur. Phys. J. D. 2007, 46, 307-313.

13. Stillinger, F. H.; Weber, T. A. Packing Structures and Transitions in Liquids and Solids. Science 1984, 225, 983-989.

14. de Broglie, L. Waves and Quanta. Nature 1923, 112, 540.

15. Mühlberger, F.; Wieser, J.; Morozov, A.; Ulrich, A.; Zimmermann, R. Single-Photon Ionization Quadrupole Mass Spectrometr with an Electron Beam Pumped Excimer Light Source. Anal. Chem. 2005, 77, 2218 2226.

16. Arps, J.; Chen, C. H.; Mccann, M. P.; Datskou, I. Ionization of Organic Molecules Using Coherent Vacuum Ultraviolet Light. Appl. Spectroc. 1989, 43, 1211-1214.

17. Hanley, L.; Edirisinghe, P. D.; Calaway, W. F.; Veryovkin, I. V.; Pellin, M. J.; Moore, J. F. $7.87 \mathrm{eV}$ Postionization of Peptides Containing Tryptophan or Derivatized with Fluorescein. App. Surf. Sci. 2006, 252, Tryptophan

18. Aicher, K. P.; Wilhelm, U.; Grotemeyer, J. Multiphoton Ionization of Molecules: A Comparison Between Femtosecond and Nanosecond Laser Pulse Ionization Efficiency. J. Am. Soc. Mass Spectrom. 1995, 6 , 1059-1068.

19. Ding, D.; Huang, J.; Compton, R. N.; Klots, C. E.; Haufler, R. E. CW Laser Ionization of C60 and C70. Phys. Rev. Lett. 1994, 73, 1084-1087.

20. Schlag, E.; Grotemeyer, J.; Levine, R. Do Large Molecules Ionize? Chem. Phys. Lett. 1992, 190, 521-527.

21. Becker, C. H.; Wu, K. J. On the Photoionization of Large Molecules. J. Am. Soc. Mass Spectrom. 1995, 6, 883-888.

22. Dey, M.; Grotemeye, J. Cluster form of Biomolecules in the Gas Phase. Eur. Mass Spectrom. 1995, 6, 95-103.

23. Kim, N. J.; Kang, H.; Jeong, G.; Kim, Y. S.; Lee, K. T.; Kim, S. K. Anomalous Fragmentation of Hydrated Clusters of DNA Base Adenine in UV Photoionization. J. Phys. Chem. A 2000, 104, 6552-6557.

24. Denifl, S.; Zappa, F.; Mähr, I.; Lecointre, J.; Probst, M.; Märk, T. D.; Scheier, P. Mass Spectrometric Investigation of Anions Formed Upon Free Electron Attachment to Nucleobase Molecules and Clusters Embedded in Superfluid Helium Droplets. Phys. Rev. Lett. 2006, 97, 043201-043204.

25. Dunbar, R. C.; Polfer, N. C.; Oomens, J. Gas-Phase Zwitterion Stabilization by a Metal Dication. J. Am. Chem. Soc. 2007, 129, 14562-14563.

26. Hu, P.; Gross, M. Gas-Phase Interaction of Transition-Metal Ions and Diand Tripeptides: A Comparison with Alkaline-Earth-Metal-Ion Interactions. J. Am. Chem. Soc. 1993, 115, 8821-8828.

27. Jurchen, J. C.; Garcia, D. E.; Williams, E. R. Gas-Phase Dissociation Pathways of Multiply Charged Peptide Clusters. J. Am. Soc. Mass Spectrom. 2003, 14, 1373-1386.

28. Ryzhov, V.; Dunbar, R. C.; Cerda, B.; Wesdemiotis, C. Cation- $\pi$ Effects in the Complexation of $\mathrm{Na}+$ and $\mathrm{K}+$ with Phe, Tyr, and Trp in the Gas Phase. J. Am. Soc. Mass Spectrom. 2000, 11, 1037-1046.

29. Schanen, P.; Yang, D.; Weinkauf, R.; Schlag, E. Efficient Cationization by Cs + Adduct Ion Formation in a Supersonic Beam. Int. J. Mass Spectrom. Ion Processes 1997, 167/168, 447-470.

30. Axelsson, J.; Scrivener, E.; Haddleton, D. M.; Derrick, P. J. Mass Discrimination Effects in an Ion Detector and Other Causes for Shifts in 
Polymer Mass Distributions Measured by Matrix-Assisted Laser Desorption/Ionization Time-of-Flight Mass Spectrometry. Macromolecules 1996, 29, 8875-8882.

31. Kale, L.; Skeel, R.; Bhandarkar, M.; Brunner, R.; Gursoy, A.; Krawetz, N.; Neal, P.; Phillips, J.; Shinozaki, A.; Varadarajan, K.; Schulten, K. NAMD2: Greater Scalability for Parallel Molecular Dynamics. J. Comp. Phys. 1999, 151, 283-312.

32. MacKerell, A. Jr.; Feig, M.; Brooks, C. III. Extending the Treatment of Backbone Energetics in Protein Force Fields: Limitations of Gas-Phase Quantum Mechanics in Reproducing Protein Conformational Distributions in Molecular Dynamics Simulations. J. Comput. Chem. 2004, 25, 1400-1445.

33. MacKerell, A. D. Jr.; Bashford, D.; Bellott, M.; Dunbrack, R. Jr.; Evanseck, J. Field, M.; Fischer, S.; Gao, J.; Guo, H.; Ha, S.; JosephMcCarthy, D.; Kuchnir, L.; Kuczera, K.; Lau, F. T. K.; Mattos, C.;
Michnick, S.; Ngo, T.; Nguyen, D. T.; Prodhom, B.; Reiher, W. E., III Roux, B.; Schlenkrich, M.; Smith, J. C.; Stote, R.; Straub, J.; Watanabe, M.; Wiorkiewicz-Kuczera, J.; Yin, D.; Karplus, M.All-Atom Empirical Potential for Molecular Modeling and Dynamics Studies of Proteins. J. Phys. Chem. B 1998, 102, 3586-3616.

34. CPMD Version 3.11.1, Copyright IBM Corp 1990-2006, Copyright MPI für Festkörperforschung Stuttgart 1997-2001.

35. Lee, S. K.; Polyakova, Y.; Row, K. H. Interrelation of Retention Factor of Amino-Acids by QSPR and Linear Regression. Bull. Korean Chem. Soc. 2003, 24, 1757-1762.

36. Kamariotis, A.; Boyarkin, O. V.; Mercier, S. R.; Beck, R. D.; Bush, M. F.; Williams, E. R.; Rizzo, T. R. Infrared Spectroscopy of Hydrated Amino Acids in the Gas Phase: Protonated and Lithiated Valine. J. Am. Chem. Soc. 2006, 128, 905-916. 